\title{
DESIGNING COMPLEX MACHINES BY A SECTIONAL ANALYSIS APPROACH: CASE STUDY THROUGH THE DESIGN OF A WATER PURIFIER
}

\author{
Samriddho Ghosh ${ }^{1^{*}}$ and Dr. Mainak Ghosh ${ }^{2^{*}}$ \\ ${ }^{1}$ Undergraduate student, Jadavpur University, INDIA, samriddho5@gmail.com \\ ${ }^{2}$ Head of Department. Dr, Jadavpur University, INDIA, mainak.ghosh@jadavpuruniversity.in \\ ${ }^{*}$ Corresponding author
}

\begin{abstract}
This article primarily discusses a design approach, in order to design a portable water purifier. It revolves around the idea of various sectional model iterations, in order to bring out the best fit product, both functionally and ergonomically. Choosing the best iteration while designing a machine, with various parts can be challenging, in case of this water purifier, consisting of various complex components, which need to be spaced in such a way to optimize both functional abilities and usability. To achieve this, the approach specifies to draw out sectional axes through the various components, through one common central point, which can be the centroid of the product. This goes for one iteration for the arrangements of components in the product. Maximum options should be laid down with the system of axes in view of the designer. Now, using a novel, intuitive approach, the best fit iteration is obtained.
\end{abstract}

Keywords: Ergonomics, functionality, product architecture, axial approach, water purifier, design thinking, modular architecture

\section{INTRODUCTION}

Down the ages, as product design evolved, it has been observed that a conflict between ergonomics, functionality has plagued designers. This is not new, since design has adapted to fit human centered use based on compromise among the factors stated. When it comes to designing a best fit product, various schools of thought have come forward placing didactics to carry to the practice forward. The set of rules, are well accommodated in producing design that is sound both ergonomically and functionally. However as designers progress the lines between these ideologies out looking various design guidelines seem to merge into a motley of approaches that govern a lot of products in the 2000s. Understanding this borderline ideology will help to shed light on why as to there exists a disparity between functionality and ergonomics. Evolutionary products since modern times have clearly delineated the design principles on which it stands-a 
great example would be the Bauhaus school of thought which propagated- 'Less is more' and 'Form over function'[1]. The products designed by the Bauhaus designers found aesthetic value in total emphasis on functionality and ergonomics.

However, down the ages it is seen that products have evoked more than a singular design foundation. This inevitably leads to different aspects of the product being designed under the veil of a mixture of design credos. Under various inventive problem solving approaches, functionality that is being catered by a specific idea can't be amalgamated to the ergonomics attributes of another [2]. This basic problem can be touted for the contradiction among the two most crucial factors of Human centered design. The problem has its inception from the very first conception stage, after which various design options are evaluated. This article aims to bolster the evaluation with a novel approach, combining both visual and analytical process flows to solve the dichotomy.

\section{RESEARCH METHODOLOGY}

The idea starts with identifying the problem- What, how and why. Understanding these three aspects will drive a designer to the bottom of the scenario. The problem being identified shall be upheld by case studies to bolster the question- As to what extent is this issue pertinent? Case studies prove the qualms of not only the designers but the users also. The analysis will uphold existing products and justify as to how the issue of functionality and ergonomics affect the entire existence of the product. The analysis will be then used as raw feed to a sample model of a portable 20 liter water purifier that will help to provide a probable solution to this exigent issue.

There will be analysis with respect to two instances- one where existing products shall be examined with respect to functionality and ergonomics, which establishes the problem statement of this article. The second analysis expresses a proposed product which is designed in a certain model that aims at solving the conundrum between the two factors. The inert manifestation of the dichotomy mentioned, leads not only to issues for designers and engineers but also contributes in a negative extent towards the user experience, for reasons pertinent to functions and how well the product can be use, which could be solved in unison. For example, various positions of the hand pump is sketched and axes are drawn along its longitudinal side- with 5 such options laid, one can select and justify which sectional position serves best in order for the user to access, use and maintain it in the most effective way. This design approach only lays before us a possibility of spatial design with regard to not only 2 dimensional but also 3 dimensional system, where the top sectional view of the iterated products have to considered. The conflict of functionality and ergonomic aspects of design can be curbed to an extent (on a strictly sectional level), by this method in order to find best fit design in terms of both the above stated factors.

\section{EXAMINING THE CONUNDRUM IN EXISTING PRODUCTS}

The nail cutter has been one of the most universal pieces of hardware to humans. With such a utilitarian approach to its design, the nail cutter has been universally accepted by the world. Although functionally adept, there lies an ergonomic concern that ails users even today- once the nail is cut very efficiently, there is no option for holding on to the cut off nail, before it falls off from the cutter mouth. A similar mechanismthe punching machine solves this problem with a polymer housing beneath the hole vice to hold on to the circular shaped paper.

This ergonomic concern might seem trivial, however this leads to inconvenience on the user end, establishing the disparity that exists between ergonomics and functionality. Such products however adept they are functionally, ergonomically might lack when it comes to user experience or vice versa. [3] On the other hand, if we take up the example of an office stapler (hand clamp type) - we find the integrated design of all components is air gapped end to end to provide a complete user experience starting off from the functional efficacy to the removal of the pin by the posterior protrusion establishing its ergonomic success as a product.

There are millions of such product that possess this issue, however few process that schedule ergonomically evaluation in tandem to functional consideration.[4] There lies factors, that affect the integration of ergonomics in design, and while adjusting to these factors, often times the other areas are difficult to compensate. There has to be an optimal approach towards this problem. 


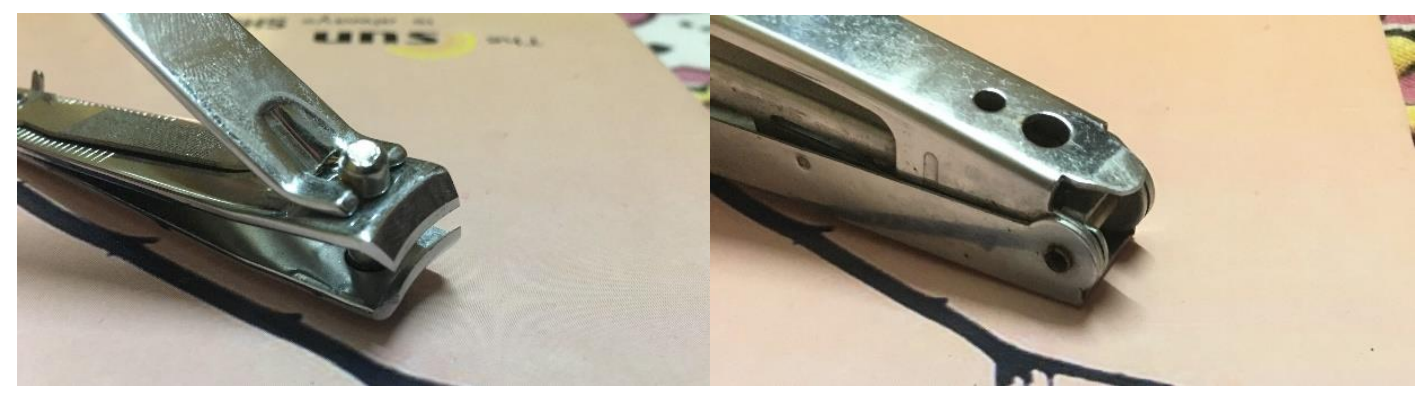

Figure 1: The cutter mouth of a nail cutter and the posterior part of the stapler used for removing staples

\section{THE AXIS MODEL - A PROPOSED METHOD TO INCORPORATE FUNCTIONALITY AND ERGONOMICS}

While designing any product, designers come up with extensive iterations to find the best case scenario that suits the problem statement of the design most aptly. However in this entire process there still exists lapses when it comes to satisfy ergonomics without harming functionality of the product or vice versa, since ergonomic design principles are extensively taught, it might be limited to certain aspects as usability, ergonomics and testing principles, however, the extensive ergonomic research data is not available readily to the designer, hence even if the functional aspect hits the bulls eye the ergonomics is seen in a vague qualitative view. [5] This leads to usage issues pertaining to the user experience.

To demonstrate the process, a proposed 20 liter non electric water purifier design is experimented upon. The purifier consists of components: the hand piston, purification system (for the sake of simplicity more complex components of the purification system is clubbed together to form one component), input opening, output tap. Alignment and positioning in each of the iterations has been done according to certain users' needs. However, it isn't possible to cater all the need based aspects for a design statement to be catered into one design iteration, it aims to approach the design process in a more targeted fashion, in order to emphasize on not only existing problems, but also issues that can arise due to the dichotomy as mentioned in earlier. Hence this process aims to club the pros from all the stated iterations and reject the cons of the iterations to come up with a best fit option.

Problem statement pertaining to the product: The problem statement requires us to design a portable, non-electric and gravity based water purifier with a capacity of 20 liters. While the water purification technology does not directly come under the purview of design, there will be instances where the water purification technology will be leveraged to bring about warranted outcomes in design. We can mainly view this as a rural product hence, focus on portability will be a major driving force in this regard. This product aims to be the inexpensive substitute to the costly electric reverse osmosis /ultraviolet water purifiers that are available at households for static use.

Problems this product aims to solve:

1. Dire consequences of prevalent bacteria, viruses and other compounds in the ground water in villages.

2. High costs of sophisticated water purification with respect to the rural society.[6]

3. Women/men in villages have to travel long distances in order to procure potable water.

4. Since water from an official supply has fixed time limits, instant need based water purification is a farce in such a rural setting.

The difference that this product brings is the fact that owing to its portability, the dynamic aspect enables it to collect water from any given source in a rural environment (hand driven tube wells, water supply taps, surface water, etc.) and purify the water on the go [7], since its gravity based. Within a standard standing time (depends on the type of feed water used) the water is ready to drink. In such a product when dealing with various components, there are certain functions that needs to be carried out in tandem with the usability aspect. This has to be ensured in order to deliver a more complete product pertaining to the longevity and the purpose of the product. For better understanding purposes, the various components of the iterations are graphically defined below, in order to leave less space for confusion when the process progresses. 


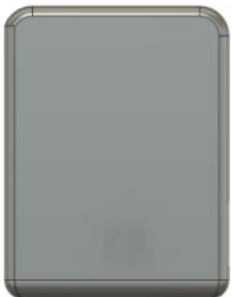

OUTER ABS SHELL

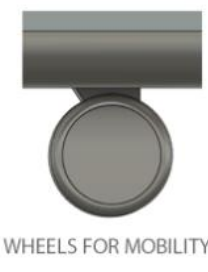

Cor

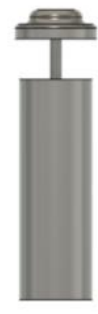

PISTON
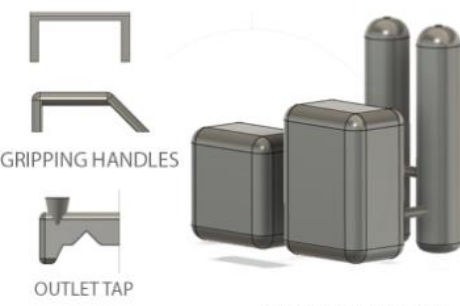

PURIFICATION UNIT

Figure 2: Various components of the Water Purifier.

In order to successfully make the components function, and make sure the user doesn't face any issues when it comes to ergonomics, the following method has been devised. The specific approach that this method undertakes, although in its nascent stage, will successfully outline, as to why the problems between functioning and the way of work in a product occurs and design thinking in which direction shall be adequate to attend this issue. These are traditional product designing methods that are employed in order to design desired outcomes, however, even among those, there are instance of missed out ergonomics or incomplete user experience. Although, such design credos are in fashion for years, but with evolving technology and need based demand, it appears to be of paramount importance that such minute detail with respect to product design should be addressed. This method aims to solve this conundrum in an axial manner that are listed as follows:

\section{Step 1- Identifying possible design iterations for the same product:}

The first step aims at guiding the entire design process onto few selected iteration from which a designer would have developed the final output. All feasible iterations need to be placed at the designer's disposal, where all components and functionalities are demonstrated in totality. This is only possible if the iterations have considered the factors of ergonomics and functionality to a certain extent. To start off, only crosssectional models of the iteration shall be used better clarity of understanding. The vertical cross sections are used at first to get the entire picture of the iteration are dealing with, rather than specific zones within the product.

First Iteration- Starting off with the first iteration, it consists of the piston and the feed water inlets places on the top surface of the purifier, at each fillet, inclined at an angle of 45 degree. The gripping handle fixated on the top surface for the purifier to be carried like a brief case.

Pros- The piston alignment enables a user to apply greater momentum with minimal force. Outlet tap placed at the optimum level of $75 \mathrm{~mm}$ above the purifier bed, to ensure no settled contaminants finds its way out via any leakage or deformity.

Cons- Owing to its capacity, the purifier with feed water inside can be heavy to carry around, (although this depends from person to person, but generally) hence just a handle for gripping purposes will not be enough to serve its portability aspect.
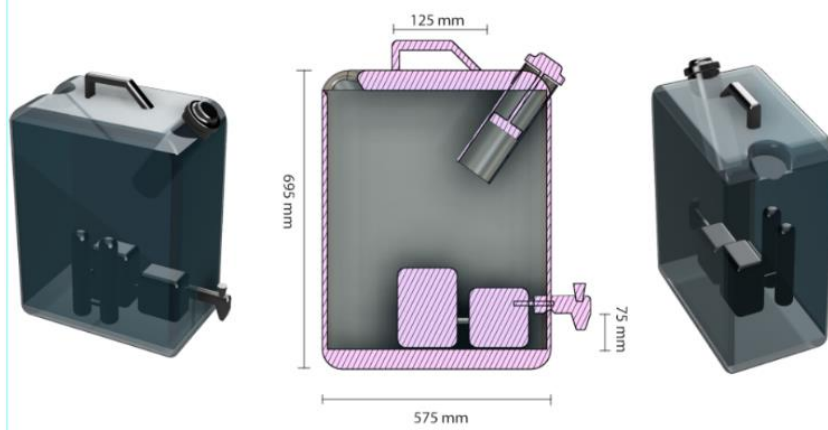

Figure 3: (From L-R) First view, Cross sectional representation and second view

Second Iteration: Here, the piston is shifted to a vertical position aligned with the feed water inlet, on the top surface of the product. The gripping handle has been placed on one of the fillet ends, pulling/pushing purposes of the purifier, especially since 4 multidirectional wheels have been attached to its base, ensures smoother mobility, negating the problem seen in the earlier iteration. These iterations are evolutionary in 
nature, however there remains certain usable aspects found in the receding design iterations that form a part of the succeeding blue prints, leading to a more coherent result.

Pros- The mouth of the inlet feed water since aligned across the horizontal axis enables, easier filling up of the purifier with feed water. The inclined gripping handle enables greater momentum for pulling or pushing the purifier on wheels. Owing to the heavy nature of the purifier, the wheels attached ensure easier and faster mobility.

Cons- The vertically aligned piston, doesn't make much difference structurally, but ergonomically is not feasible owing to its directionality. Visually or functionally this is a trivial issue, but if the product turns out difficult to operate, mentally that's a deterrent against widespread acceptability and success of a particular product.
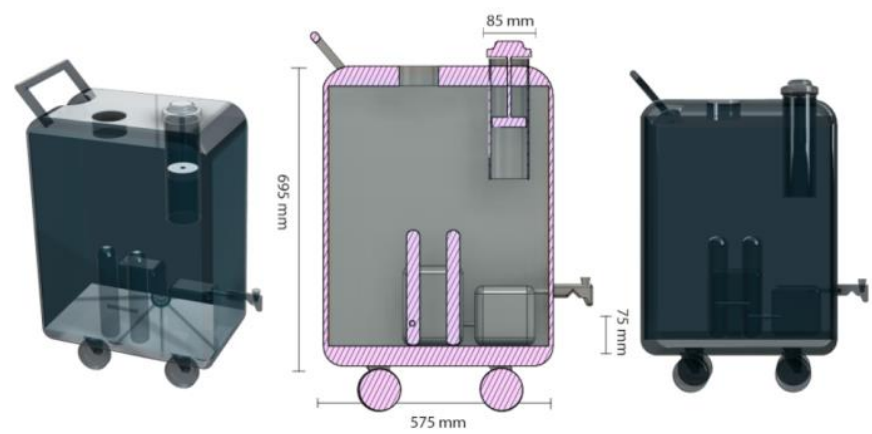

Figure 4: (From L-R) First view, Cross sectional representation and front view

Third Iteration: In this last iteration, not much functional changes has been down, but few ergonomic changes are in view. The piston has been shifted to the side of the purifier with its axis aligned along the horizontal axis. The inlet hole has been at the same place since the second iteration. The gripping handle has been shifted to the top horizontal surface of the purifier. This has been done since due to the multi directional wheels, it is really trivial as to how the purifier is pushed, but still its ergonomic aspect will be inspected below.

Pros and Cons- This clubs both the positives and the negatives because this is a more of an repetitive iteration when it comes to functionality however, there are ergonomic differences like the horizontal piston, which shall not be adept enough in perturbing the feed water through the purification unit due to differential force distribution owing to the 90 degrees formed between the axis of the piston shaft and the direction of acceleration due to gravity.
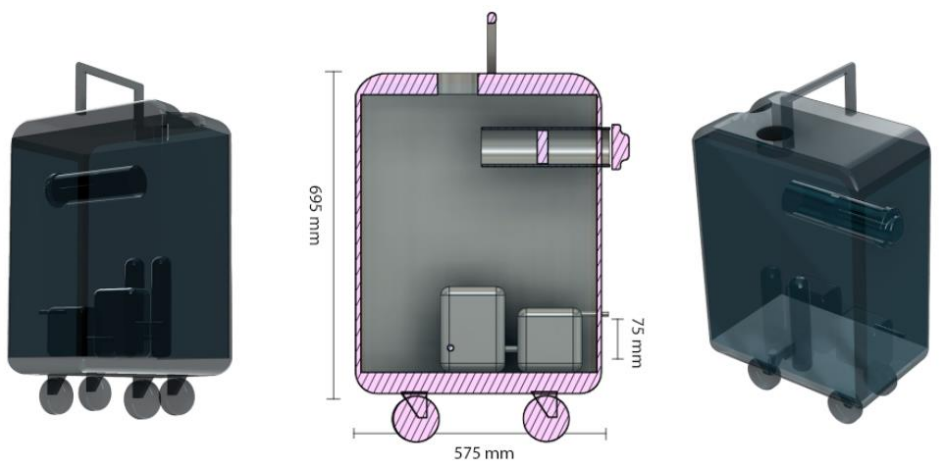

Figure 5: (From L-R) First view, Cross sectional representation and second view

\section{Step 2- Drawing axes:}

This is the step where the designer needs to identify each and every component of the product as placed (from the cross-sectional drawings of the iterations) keeping various factors in mind. Now, cross-sectional axes are to be drawn a across each component in a direction that seems feasible to the designer (the designer needs to identify a visual direction for each component in order to draw the axis).

This process is to be repetitive for every iteration till the point we have various cross-sectional drawings of 
iterations with their individual component axes laid out. Things are obscure till this part since the axes will make more sense in the later stages of the process. In very complex machines where intricate components meddle with the axes of one another, they can be grouped together to form a congenial axis in order to place that group of components together. The entire point of this exercise is to point out not only the components but also various poisoning and directionalities of the components.

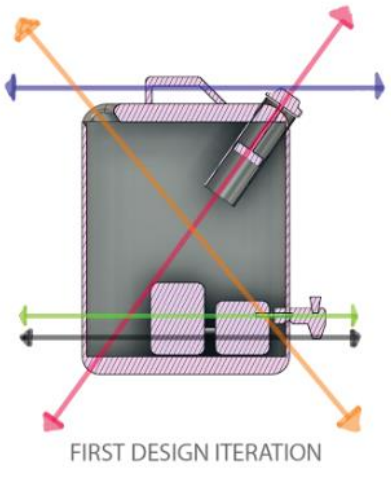

Figure 6: Axes drawn across every component in each iteration

In figure 6, the blue axis denotes the axis of the gripping handle, the orange axis stands for feed water inlet opening, while the red line represents the piston axis, the green line denotes the axis for the outlet tap and lastly, the black axis denotes the axis of the purification unit.

The color notation has been done in order to identify the disparate component positions and their directions in a given design iteration.

\section{Step 3- Blurring the background:}

Now with the axes drawn on the sectional drawings of the iterations, the designer needs to remove/blur/erase the sectional drawings in the background to bring out the axes marked on it. The diagram below depicts the situation as stated. This brings on to the table the various options in terms of arrangements of components that are iterated according to a certain degree of functionality and ergonomics. The schematics of each iteration represented by only the axes provide a sense of the spatial extent to which the particular iteration extends to. This was the outcome if inspected on a whole to part approach, however if scrutinized from a part to whole approach this method signifies internal spatial distribution of each component in a certain region of the iteration. For example:

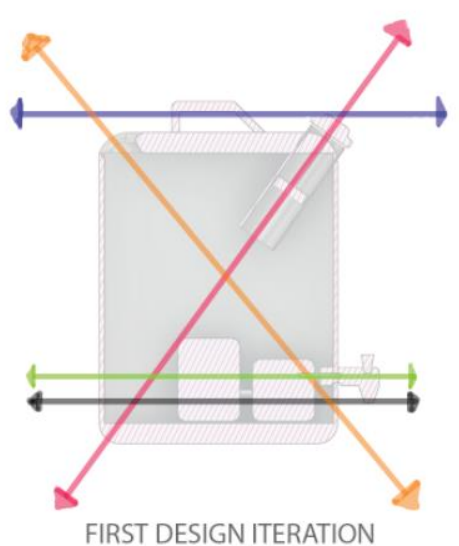

FIRST DESIGN ITERATION
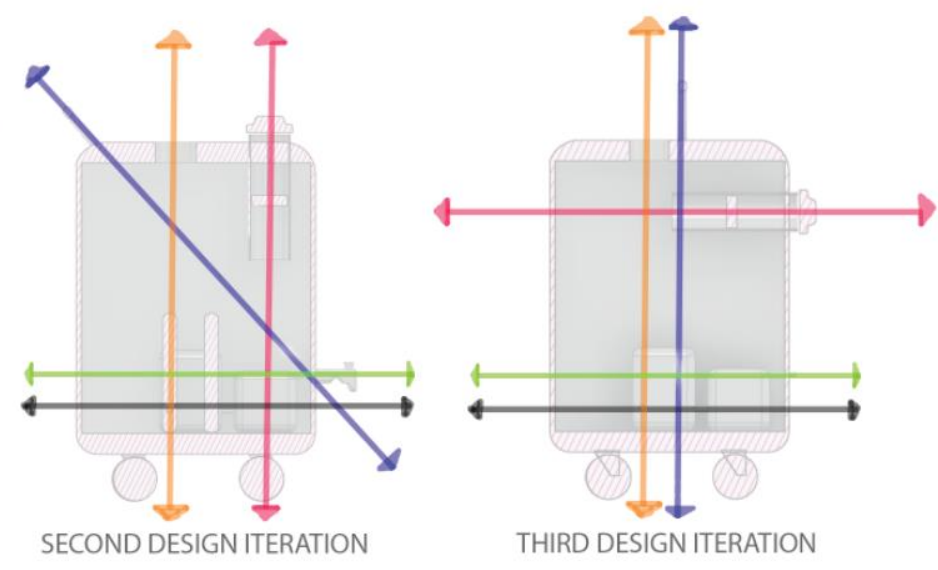

Figure 7: Various axis implementation for the design iterations

In these sectional models, now we can understand the relative position of a component with respect to its environment. This enable us to understand that positioning since it's tied to both ergonomics and functionality, can be a deciding factor while designing complex mechanisms as such.

\section{Step 4: Delineating spatial zones with regard to each component:}


Without the actual representation of the components but only the axes, the designer is to mark spatial zones with regard to each axis, adding 2 dimensional weightage to the otherwise skeletal axis system. This is a purely quantitative and intuitive exercise unlike the other steps. It is preferable to render each zones with a separate hue to understand the identity of each zone corresponding to their given axis, representing each component.

Now considering a single components, the spatial zone designates the extent of its position in order to cater to a specific functionality. Now, having all such zones for all such iterations, will help us understand where and how such components are placed to solve specific problems pertaining to each iteration. With this knowledge in hand, the next step pertains to conglomerating the three axis models into one and adjudging a qualitative average of the various component positions to come up with the final outcome of all the various iterations.
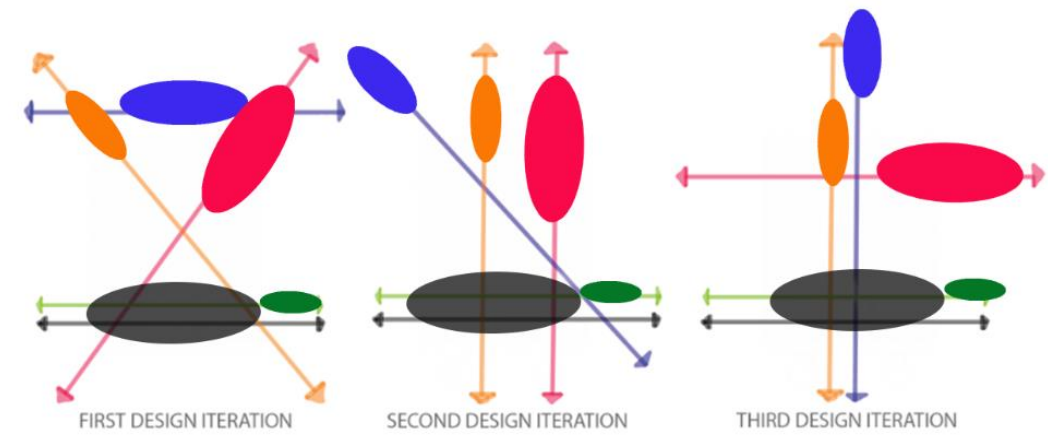

Figure 8: Various spatial zones with regard to the components and their axes

Since these spatial zones are component specific, these act as indicators for a holistic understand of the relation between positioning and ergonomics. The spatial zones are seen maybe only in this step, as we move forward. Hence, this step marks the axes, zoning and directionality into one picture as shown in figure 8. Once we start conglomerating all given iterations we shall find how a relative position of a component is delocalized into a broader zone within the product marking a broader periphery within which all such possible iterations of a specific component is located.

Questions may arise, whether the same procedure is applicable for the outer ABS shell or any other casing or the wheels. While for any other casing, it depends on the positioning of the components it aims to cover, hence, both the external and internal component shall be taken care of, while the same model can be outlined on the outer ABS shell to demarcate its apt form.

\section{Step 5- Conglomerating all axes:}

This is the step where things start to come together. Earlier, the design as demarcated all axes and spatial zones and developed a holistic view of the component structuring in the product. Now this dispersed view of multiple iterative structures, needs to be gathered together into one form. The form development pertaining to the final product starts from this stage.

This process can be labelled as an intuitive and cognitive process, where designing and critical thinking plays an important role, as we move forward.

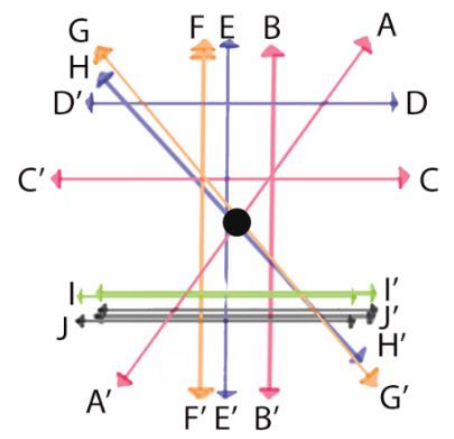

Figure 9: Conglomerated axes model

The black spot in the middle represents a single point on which the three axes models are pivoted. This specific point shall serve as the central point of the final outcome. Figure 9 summarizes all developments 
shown in figure 6- figure 8 , on the other hand the figure 9 , forms the first rough axes model of the final design. Every axis shown in figure 9 represents each component- hence, all relative positioning of the components with respect to one another is intuitively understood by the designer/engineer. Now, this knowledge will ultimately facilitate the final positioning of the components, to an desire d outcome in step 6 , where the conglomeration of all the steps in the previous parts, shall reap an outcome, that shall comply to the best extent, with regard to maintaining a balance between the extent of functions and the way the product shall facilitate the user.

\section{Step 6 - Finding the middle ground for the final product:}

With the conglomerated axes model in view, it's time to come up with a qualitative understanding of the final element placement within the casing. From figure 8, the spatial convergence of each component in all three iterations are to be figured out and then, we have to chalk out a similar representation as that of figure 8 , displaying the spaces with maximum density of each component, coming up with a 'visual average' for the qualitative approach.
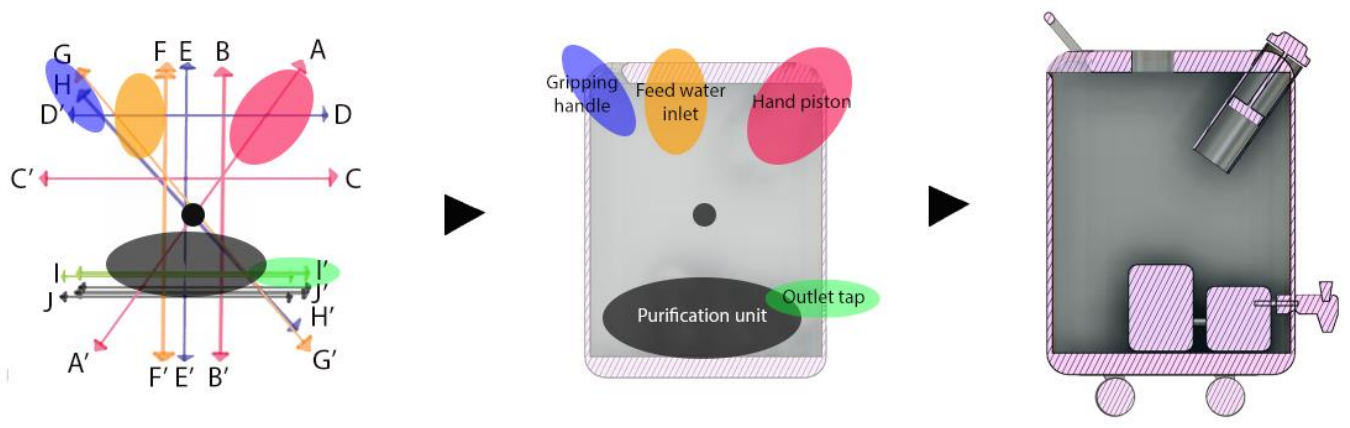

Figure 10: (Form L-R) Spatial convergence diagram, final zoning, and proposed section of the outcome

After the final 2D spatial distribution is formed, we now have a clear picture with respect to the zoning of the components in the final iterative design. The next step takes requires the designer to form the last and final iterative design based on this zoning. The directionality reference of each component which initially invoked as a perceptual experience first on the part of the designer which is resonated through the product to the user [8] might be taken from the diagram form the earlier steps, however the gross placement of the elements inside the ABS shell can be formed.
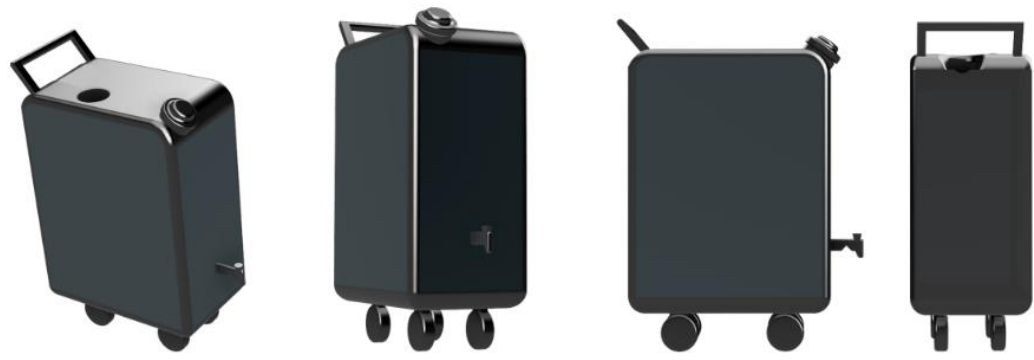

Figure 11: (L-R) First view, second view, front elevation, side elevation of the final iteration

Whether the final outcome shown in figure 11, complies with the objective of functional and ergonomical optimization, it can be matched with the solutions of the problems the designer tried to solve in step 1 , starting with the angle and placement of the piston, to whether the gripping handle shall be upright or inclined with regard to momentum generation and also user friendliness. The outcome we are dealing with can be arrived via the other methods that have been used all this while, but since this process specifically focuses on ergonomic and functional concerns, it holds more grounds, pertaining to these specific factors. Iterating the features to find the best fit product is the commonplace approach to specifying product architecture, but this method establishes ground to justify the concerns relating to future issues faced during the lifetime of the product.

\section{Summarizing the process:}

The individual axial approach that is purported in the process is a simple manifestation of conglomerating all 
possible sectional positions of each component in a product. This helps the designer or the team to get the entire view of options available into one single methodical diagram, which can be supported by logical reasoning and not just intuition of the designer or the team. Intuition plays a major role in such product developmental process, but however, the final stages required logical apprehension of the decisions taken by the designer with regard to every aspect of the product. To bridge the gap between the intuitive approach of the design thinking process and the logical diagrammatic approach, this axis model can play a fundamental role.

Looking at the entire process in a nutshell, the simplification of arrangement and combination of complex parts within a machine or a product, this approach empowers the designer to avoid missing out certain iterative advantages that are condoned on the very occasion of selecting a best fit option according to the requirement

This process is primarily dependent on the visual and the analytical implementation of various case wise scenarios in both high fidelity and low fidelity prototyping processes. The under lying idea governing this implementation is manifested from the cognitive sense of the designer that is purported through analytical backing for every choice that is made in this particular design process. The assumption or the prerequisite with which this process proceeds takes in to account that the sectional iterations presented to the model, have been curated with the considered of functionality and ergonomics to the best extent. This requirement allows the model to optimize each iteration in order to produce a best case possibility, otherwise it is graphically or visually improbable to ascertain various functional and ergonomical advantages in every iteration the designer or the team comes up with. With regard to the entire product development process, this approach can be implemented in the transition of the rough System level design to the detailed geometric design. The process plan that is established at the end of the process flow shall have critical issues considered throughout the development process, solving which becomes a primary objective of the axial approach proposed in the article.

\section{Relation with product architecture:}

In relation to the two distinct types of product architecture, one being modular and the other being integral, we find the application of the above stated axes method in primarily integral product architecture. There are many factors that motivate the product architecture, in this case it's the ergonomics. A product that imbibes integral product architecture shall be designed keeping the high possible performance into consideration. However there are two issues that still find its place when the axes model is seen in the light of product architecture:

Functional components maybe distributed across multiple spaces and boundaries between such spaces are difficult to delineate. (Step 4 aims to resolve this issue)

Modifications with respect to one or a few components can lead to a complete redesign of the existing product. Now these two issues are addressed in the steps where individual component zones are marked to get a rough outline of the extent of the sectional positioning of a particular functional component. Therefore, for cases where modifications are to be made, the axes model can be helpful in mitigating the problem, by identifying the components and understanding their relative position and a decision can be taken by judging the spatial context as to whether a complete redesign is required or not. However, no product can be declared as strictly modular or integral in its architectural context, hence it is safe to assume that such a model will also find its usability in modular products, especially in cases of slot modular and sectional architecture [9] as showed in figure 12.
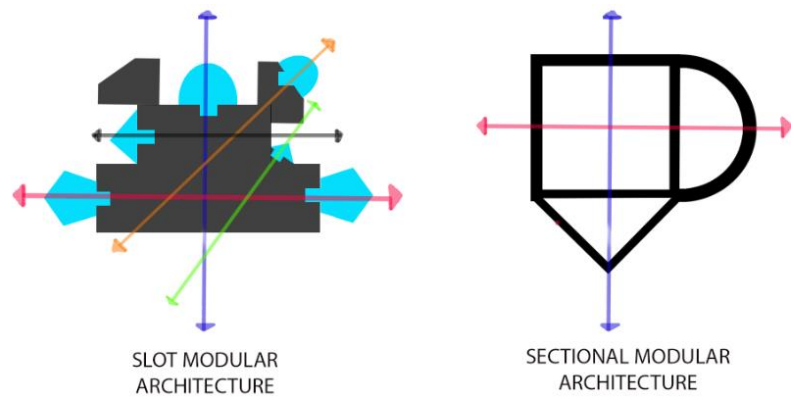

Figure 12: Axes on various components of different types of product architecture

Drawing reference from the guidelines for establishing product architecture [10] - this axes model finds its acceptance as a transitional process when moving form creating a preliminary geometric layout (2D or 3D) to 
identifying the incidental and functional interaction among the components. Layout decision criteria are closely knit with to the various clustering issues, the designer might find that a certain spatial allocation done in step 4 might not be geometrically feasible, which leads to reassigning of elements. The outcome of the process of the Axes model can be used as reference by Industrial designers for understanding and solving the issues pertaining to aesthetics and human-machine interaction. This marks the present and the futuristic pedestal on which we can find the application of such a model. Bolstering with data that is applicable in the axis model, it shall serve as a new dimension of intuitive designing through a visual procedure. The choice of product architecture has broad revelations when it comes to product performance, variety and manufacturability. Selecting the proper architecture is pivotal during the concept development phase and system level design phase, to provide valuable input into structure of the problem that needs to be solved and the impedance that is faced while reaching the desired goal. The method stated in the article shall help in the integration analysis to define not only the product architecture but also the physical structural details.

\section{CONCLUSION}

This article starts off by citing the issues in products that have plagued designers and users alike- the article establishes a proposed method to accommodate every computational aspects of both function and ergonomics in order to come up with more adroit products in totality. In this process the steps outlines fit into a specific place in the product architecture chain as mentioned in the earlier section where this axes model finds its usability in the concept stage of the design process, where decisions taken on the basis of this theory can alter designs, manufacturing processes or rather how users view a product in an overall scenario. To substantiate this process an example of a portable water purifier was used that contained, ergonomic and functional flaws in its iterations, instead of choosing any one form the said option, the axes model outlines a way to conglomerate the pros of the iterations to come up with another iteration that imbibes the best of all the other iteration, to come up with a better option, in terms of both the factors.

\section{REFERENCE LIST}

[1]- Chen, Wenwen \& He, Zhuozuo. (2013). The Analysis of the Influence and Inspiration of the Bauhaus on Contemporary Design and Education. Engineering. 05. 323-328. 10.4236/eng.2013.54044.

[2]- Ghimisi, Stefan \& Dana, Nicula. (2017). PRODUCT DESIGN PRINCIPLES.

[3]- Tosi F. (2020) Ergonomics and Design. In: Design for Ergonomics. Springer Series in Design and Innovation, vol 2. Springer, Cham

[4]- Högberg, Dan. (2005). Ergonomics Integration and User Diversity in Product Design.

[5]- Luximon Y., Luximon A., Fu F. (2019) Rethinking Ergonomics in Design. In: Goonetilleke R., Karwowski W. (eds) Advances in Physical Ergonomics \& Human Factors. AHFE 2018. Advances in Intelligent Systems and Computing, vol 789. Springer, Cham

[6]- Jain S.K., Agarwal P.K., Singh V.P. (2007) Problems Related to Water Resources Management in India. In: Hydrology and Water Resources of India. Water Science and Technology Library, vol 57. Springer, Dordrecht

[7]- Taheran, M., Kumar, P., Naghdi, M. et al. Development of an advanced multifunctional portable water purifier. Nanotechnol. Environ. Eng. 4, 7 (2019).

[8]- Tosi F. (2020) From User-Centred Design to Human-Centred Design and the User Experience. In: Design for Ergonomics. Springer Series in Design and Innovation, vol 2. Springer, Cham

[9]- Ulrich, Karl T, and Steven D. Eppinger. Product Design and Development. (7th Edition), 2008. Print.

[10]- Thomas U. Pimmler, Steven D. Eppinger (1994) Integration Analysis in Product Decomposition, Massachusetts Institute of Technology Cambridge, Massachusetts 